\title{
Palmitopamba: yumbos e incas en el bosque tropical al noroeste de Quito (Ecuador)
}

Palmitopamba: Yumbos et Incas dans la forêt tropicale du Nord Ouest de Quito

(Équateur)

Palmitopamba: Yumbos and Incas in the tropical forest northwest of Quito

(Ecuador)

Ronald D. Lippi y Alejandra M. Gudiño

\section{OpenEdition}

Journals

Edición electrónica

URL: http://journals.openedition.org/bifea/1842

DOI: $10.4000 /$ bifea. 1842

ISSN: 2076-5827

\section{Editor}

Institut Français d'Études Andines

\section{Edición impresa}

Fecha de publicación: 1 diciembre 2010

Paginación: 623-640

ISSN: 0303-7495

\section{Referencia electrónica}

Ronald D. Lippi y Alejandra M. Gudiño, « Palmitopamba: yumbos e incas en el bosque tropical al noroeste de Quito (Ecuador) », Bulletin de l'Institut français d'études andines [En línea], 39 (3)| 2010, Publicado el 01 junio 2011, consultado el 07 diciembre 2020. URL : http://journals.openedition.org/ bifea/1842 ; DOI : https://doi.org/10.4000/bifea.1842

Les contenus du Bulletin de l'Institut français d'études andines sont mis à disposition selon les termes de la licence Creative Commons Attribution - Pas d'Utilisation Commerciale - Pas de Modification 4.0 International. 


\title{
Palmitopamba: yumbos e incas en el bosque tropical al noroeste de Quito (Ecuador)
}

\author{
Ronald D. Lippi* \\ Alejandra M. Gudiño**
}

\section{Resumen}

Las investigaciones hechas en Palmitopamba, en la montaña occidental de la provincia del Pichincha (Ecuador), revelan un centro monumental ocupado durante varios siglos por los yumbos, población nativa de la zona. La llegada de los incas en esta región a eso de 1500 d. C. permite estudiar la relación que tuvieron con este cacicazgo ubicado en una zona de bosque tropical húmedo. La expansión del Tahuantinsuyu parece haber tomado un rumbo distinto en el país de los yumbos. El artículo presenta varias interpretaciones sobre la importancia del sitio tanto para los yumbos como para los invasores desde las perspectivas de la arqueología del paisaje y de la etnohistoria. Brinda también un estudio de esta interacción después de la conquista española en 1532 y del posible papel del sitio en un levantamiento indígena fracasado.

Palabras claves: El Ecuador, incas, yumbos, bosque tropical, Periodo Tardío

Professor of Anthropology, University of Wisconsin-Marathon County, 518 S. $7^{\text {th }}$ Avenue, Wausau, WI 54401, USA. E-mail: ronald.lippi@uwc.edu

** Human Environmental Sciences, University of Missouri-Extension, 1205 University Ave, Suite 1100, Columbia, MO 65211, USA. E-mail: gudinoa@missouri.edu 


\title{
Palmitopamba : Yumbos et Incas dans la forêt tropicale du Nord Ouest de Quito (Équateur)
}

\section{Résumé}

Les recherches effectuées sur le site de Palmitopamba, dans les montagnes occidentales de la province du Pichincha (Équateur) ont mis à jour un centre monumental qui fut occupé pendant des siècles par les Yumbos, natifs de cette région. L'arrivée des Incas, vers 1500 ap. J.-C, nous permet d'étudier leurs rapports avec cette population de la forêt tropicale humide. L'expansion du Tahuantinsuyu semble avoir pris un tournant différent chez les Yumbos. Cet article présente diverses interprétations sur l'importance du site, autant pour les Yumbos que pour les envahisseurs, du point de vue de l'archéologie du paysage et de l'ethnohistoire. Ils étudie également cette interaction après la conquête espagnole en 1532 et le rôle possible du site au cours d'une tentative manquée de soulèvement indigène.

Mots clés : Équateur, Incas, Yumbos, forêt tropicale, Période Récente

\section{Palmitopamba: Yumbos and Incas in the tropical forest northwest of Quito (Ecuador)}

\begin{abstract}
Research at the Palmitopamba site in the western montaña of Pichincha province (Ecuador) has uncovered a monumental center occupied for several centuries by the Yumbos, the natives of the zone. The arrival of the Incas in this region around A.D.1500 allows us to study the relationship between the Incas and this tropical forest chiefdom. The expansion of Tahuantinsuyu seems to have taken a distinct course in Yumbo country. This paper presents some interpretations on the importance of the site for the Yumbos as well as for the invaders from the perspectives of landscape archaeology and ethnohistory. It also studies the fate of this interaction after the Spanish conquest of 1532 and the possible role of the site in a failed indigenous uprising.
\end{abstract}

Key words: Ecuador, Incas, Yumbos, tropical forest, Late Period

\section{EL PAÍS YUMBO}

Quito, capital del Ecuador, ciudad a la vez antigua y moderna de un millón y medio de habitantes, se ubica al pie del volcán Pichincha en un valle interandino, aproximadamente a 2900 metros sobre el nivel del mar. Al oeste del Pichincha y de los volcanes que forman la Cordillera Occidental, se encuentra el País Yumbo que se extiende desde las bocas de montaña hasta el pie de los Andes y el comienzo de la llanura costera. El contraste del paisaje entre el valle de Quito ubicado en la sierra y el País Yumbo en la selva húmeda es impresionante. Hasta finales del siglo XX, el área que hoy consideramos el País Yumbo se encuentra despoblada y casi abandonada debido en parte a su muy difícil topografía, al espesor de la vegetación, y a la falta de buenas carreteras.

En 1984 Lippi comenzó una exploración arqueológica de la zona por medio del Proyecto Pichincha Occidental, cuyos resultados se presentaron en varios 
artículos y dos libros (Lippi 1998; 2004a). Al principio se conocía muy poco sobre los yumbos que fueron los pobladores autóctonos del área en los últimos siglos antes de la llegada de los españoles. La investigación etnohistórica realizada por Salomon (1997) constituyó uno de los muy pocos referentes sobre esta población. Gracias a su trabajo supimos que los yumbos se organizaron en una serie de cacicazgos en el bosque tropical, que vivían en pueblos dispersos en chozas de caña de guadúa y hojas, y que mantenían un comercio muy importante con los señoríos de la sierra circunquiteña.

Por medio de las prospecciones a nivel regional realizadas por Lippi y también por excavaciones que él llevó a cabo en el sitio de Nambillo en la parroquia de Mindo, se pudo construir una cronología tentativa cultural de la región, en la cual los yumbos aparecieron en el Periodo Tardío (a veces mal llamado el «Periodo de Integración» en el Ecuador) alrededor de 1000 d. C. Los yumbos hablaban un idioma ya desaparecido de la familia Barbacoa, relacionado con los idiomas de los caranquis, los pastos, y de otros grupos en el norte del Ecuador y sur de Colombia. En base a análisis de estudios lingüísticos y la información arqueológica y etnohistórica existente, Lippi (2004b) propuso la hipótesis según la cual los yumbos se separaron de los caranquis y de los panzaleos de la sierra y fueron a vivir en el bosque tropical de Pichincha. Estudiando la distribución de cerámica Yumbo y de las tolas` rectangulares con plataforma, Lippi $(1998 ; 2004 a)$ delimitó tentativamente el País Yumbo. Cabe mencionar que estas conclusiones e hipótesis serán modificadas mientras avancen los estudios nuestros y de otros investigadores en la zona. En los primeros siglos después de la conquista española, los yumbos prácticamente desaparecieron debido principalmente a epidemias (Lippi, 2001; resumen breve en Lippi, 2004a) hasta permanecer en el olvido de la conciencia del Ecuador contemporáneo. Los tsáchilas (colorados) de Sto. Domingo hasta cierto punto son los últimos descendientes de los yumbos y probablemente de otras etnias de los bosques occidentales.

\section{EL SITIO DE PALMITOPAMBA}

Lippi descubrió el sitio de Palmitopamba en 1984 durante la prospección regional de la zona occidental de la provincia del Pichincha. Palmitopamba es un pueblo de la parroquia de Nanegal, ubicado aproximadamente a $45 \mathrm{~km}$ al noroeste de Quito. El sitio que estudiamos es muy extenso, pero los trabajos arqueológicos se han concentrado en una loma muy alta y empinada a $1480 \mathrm{~m}$ sobre el nivel del mar. Como en la mayor parte del País Yumbo, la zona está cubierta por un bosque húmedo tropical. En 1984 la parte más alta de la loma ubicada directamente al sur del pueblo era un potrero y se vislumbraban algunas terrazas o plataformas en la ladera. Lippi ha podido comprobar la existencia de algunas terrazas artificiales, muros de retención y la presencia de cerámica Yumbo e Inca

1 Las «tolas» son los montículos artificiales de tierra construidos en el Ecuador prehispánico. 
durante la prospección. Por lo tanto Lippi registró el sitio como el «Pucaráz de Palmitopamba» (NL-20) reflejando así su interpretación preliminar: se trata de un fuerte Inca en plena zona tropical.

Temporadas posteriores de excavación permitieron comprobar que el sitio fue un puesto de control militar de los incas. Sin embargo, descubimos además que fue ocupado durante varios siglos por los yumbos antes de la llegada de los incas y que fueron los yumbos, y no los incas, quienes modificaron la loma para crear un centro monumental. Por lo tanto, ya no lo llamamos el «Pucará de Palmitopamba» sino simplemente «Palmitopamba».

En la figura 1 se puede apreciar una vista de la loma desde el pueblo con las terrazas apenas visibles en el potrero, que corresponde al «Área A» donde se ha realizado hasta ahora la mayoría de las excavaciones. Cabe anotar aquí que a pesar de la deforestación de la zona durante las últimas décadas, la parte de atrás del sitio está cubierta todavía de bosque tropical muy tupido.

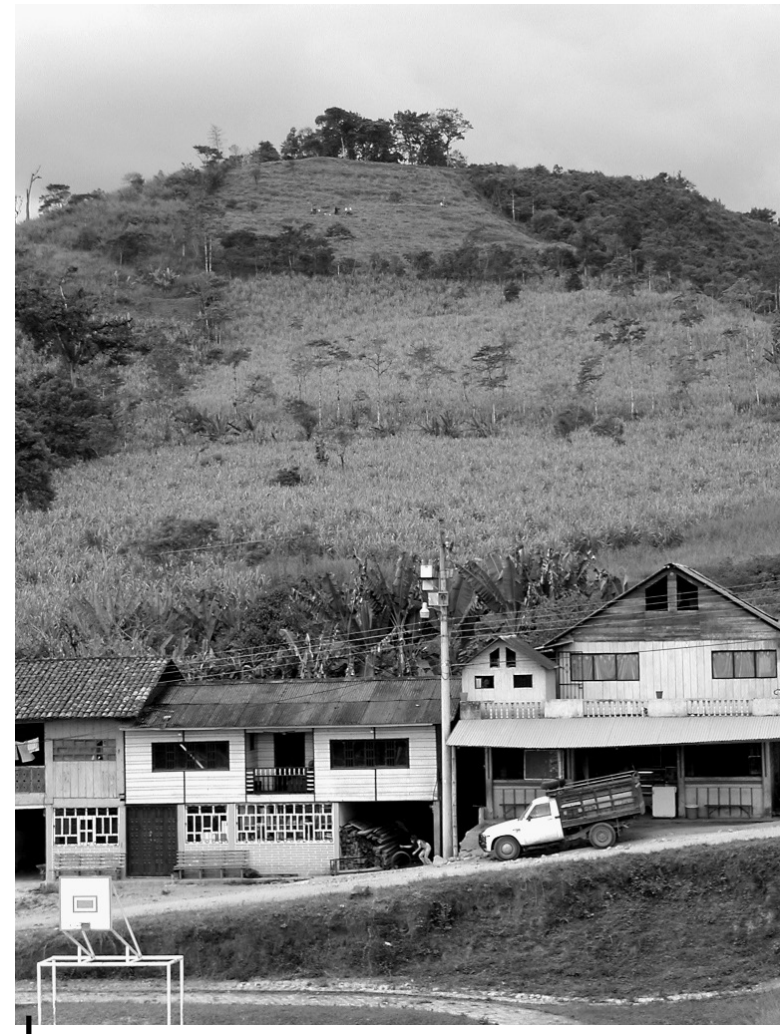

Figura 1 - El sitio de Palmitopamba (NL-20) desde el pueblo

En el potrero arriba en la loma (designado «Área A») están apenas visibles las terrazas y el equipo arqueológico
Las investigaciones en el sitio de Palmitopamba siguen adelante y los análisis aún se encuentran incompletos. Este artículo es un resumen de los hallazgos principales hasta el presente (2009) y debe ser considerado provisional.

\section{EXCAVACIONES TEMPRANAS EN PALMITOPAMBA}

En 2002 Lippi y Tamara Bray realizaron junto con varios estudiantes y trabajadores locales, una serie de sondeos en las Terrazas 1 a 6 (Terraza 1 es la cima de la loma). Las sorpresas fueron inmediatas. Primero, se pudo determinar que, a pesar de ser esta loma muy alta, los yumbos depositaron encima de ésta al menos tres metros de relleno creando una plataforma horizontal. Esto se pudo confirmar gracias a los sondeos de $1 \mathrm{~m} \times 1 \mathrm{~m}$ que llegaron a $3 \mathrm{~m}$ de profundidad sin poder

2 «Pucara» o en el Ecuador «pucará» es palabra quechua (idioma de los incas) para una fortaleza, especialmente de construcción Inca. 
alcanzar la superficie antigua de la loma. Esta plataforma tenía una apariencia bastante plana y contaba con taludes paralelos que daban la apariencia de un rectángulo. Debido a la erosión y a un desplome, ya no se conserva muy bien la forma rectangular, pero es evidente que los yumbos construyeron encima de la loma una tola rectangular con plataforma. Se cree, como parece ser el caso para todas las tolas rectangulares con plataforma en el País Yumbo, así como en el País Caranqui de la Sierra Norte, que esta tola servía como base para la vivienda de un cacique o de un templo u otra construcción de uso ceremonial y/o político.

La segunda sorpresa fue el descubrimiento de una delgada capa de tefra volcánica en algunos sondeos que se hicieron en las terrazas. A pesar de ser una loma muy empinada, las capas volcánicas formaban un estrato horizontal, lo cual indicaría que la ceniza volcánica cayó después de la construcción de las terrazas. Los vulcanólogos Patricia Mothes y Pete Hall de la Escuela Politécnica Nacional en Quito determinaron que la ceniza pertenecía al Volcán Quilotoa, el cual experimentó una enorme erupción alrededor de 1100 ó 1200 d. C. Se asumió que las terrazas, que prácticamente son desconocidas en el norte del Ecuador, eran una construcción Inca. Sin embargo, la evidencia tefravolcánica se mostró contundente: las terrazas fueron construidas al menos tres siglos antes de la llegada de los incas al norte del Ecuador, la cual ocurrió alrededor de 1490. Es decir, los yumbos, considerados por los incas como «indios desnudos, pobres y de poco valor» (Cabello Balboa, 1951 [1586]: 437-438) contaban con una organización sociopolítica suficiente como para realizar obras monumentales en la selva.

Desde la cima del sitio de Palmitopamba, la vista es magnífica y estratégica. Se puede observar una serie de tolas hacia el oeste y el norte, mayormente de forma rectangular con plataforma y rampa, ubicadas en los filos de las montañas. Palmitopamba parece ser la tola más alta de todas. Desde Palmitopamba se ve fácilmente el flanco occidental del País Caranqui al otro lado del Río Guayllabamba, y en días despejados, se ve el volcán Cotacachi, un cerro muy importante para los cacicazgos Caranquis de la Sierra Norte. También desde Palmitopamba se ve el volcán Pichincha en cuya falda oriental se encuentra Quito. Es decir, la tola de Palmitopamba permite un reconocimiento, un acceso visual, y un control no solo a una parte del País Yumbo sino también de los cerros claves del País Caranqui y de los señoríos de la zona de Quito. Su ubicación geográfica es ideal y, por lo tanto, lo consideramos un sitio muy importante en la región.

Los sondeos en la cima de la loma dieron como resultado solamente cerámica Yumbo y la presencia de arsenales de bolas de honda Inca ubicadas en el talud de la tola (fig. 2). Las excavaciones revelaron que estos depósitos fueron la última línea de defensa de los incas. Es muy posible que estos arsenales fueran más simbólicos que tácticos, quizás una manera de señalar el control Inca sobre la loma. No se descubrió ninguna construcción Inca, lo cual parece apoyar nuestra interpretación de que la cima fue una tola, es decir una huacaz, de los yumbos y que fue respetada por los invasores del Tahuantinsuyu. 


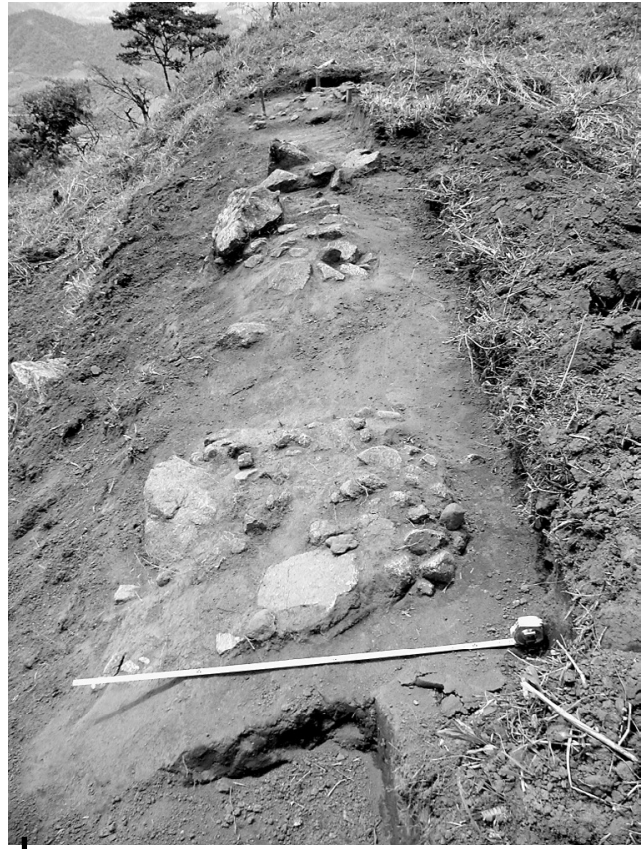

Figura 2 - Depósitos circulares de piedras de honda en la cima de la loma, que corresponde a la plataforma superior de la tola
Sin embargo, mientras se bajaba de la primera terraza, es decir la tola, y se comenzaba a excavar las terrazas más bajas, empezó a aparecer cerámica Inca entremezclada estratigráficamente hasta cierto punto ${ }^{4}$ con la cerámica Yumbo. También se descubrieron algunas pequeñas construcciones Incas de piedra. Entre estas construcciones destacaron en la Terraza 3 un muro de contención (fig. 3), un círculo de piedras de dos metros de diámetro que no era un amontonamiento descuidado de rocas sino un círculo casi plano esmeradamente elaborado, y dos círculos más de piedras pero de menor diámetro. Aún no se excavó debajo de los círculos de piedra. A pocos metros del círculo más grande se descubrió lo que parecía ser el comienzo de otro muro Inca. Era una línea de piedras labradas en una cara y con un largo de aproximadamente 10 metros (fig. 4). Tenía todas las características de un muro Inca pero presentaba una sola cara y sin relleno en la mitad. Es decir, parecía un proyecto incompleto.

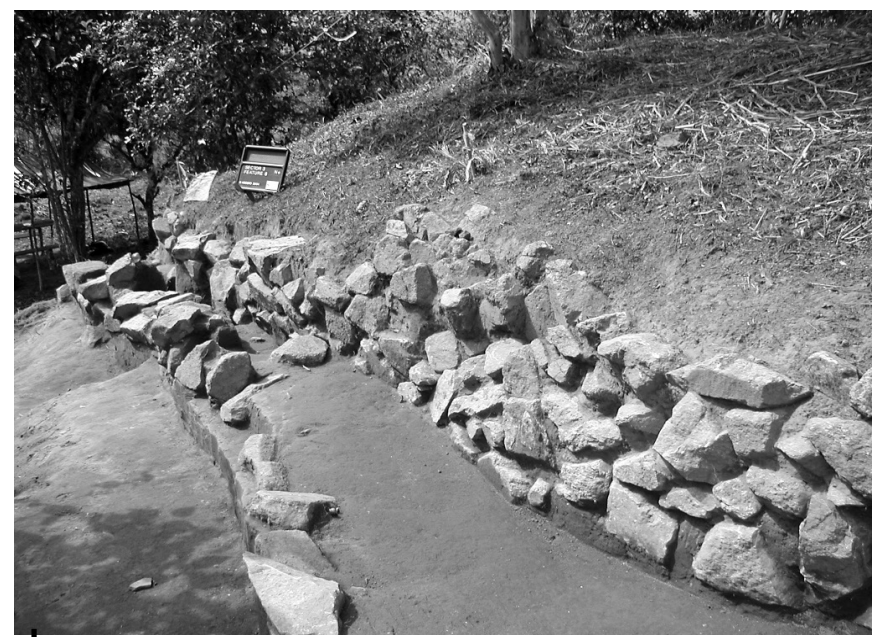

Figura 3 - Un muro de retención (no reconstruido) hecho por los incas en el talud occidental de la Terraza 3

La Terraza 4 se ve a la izquierda y al fondo

4 Hasta $40 \mathrm{~cm}$ de profundidad, más o menos, y luego más adentro solamente se encontraba alfarería Yumbo. 


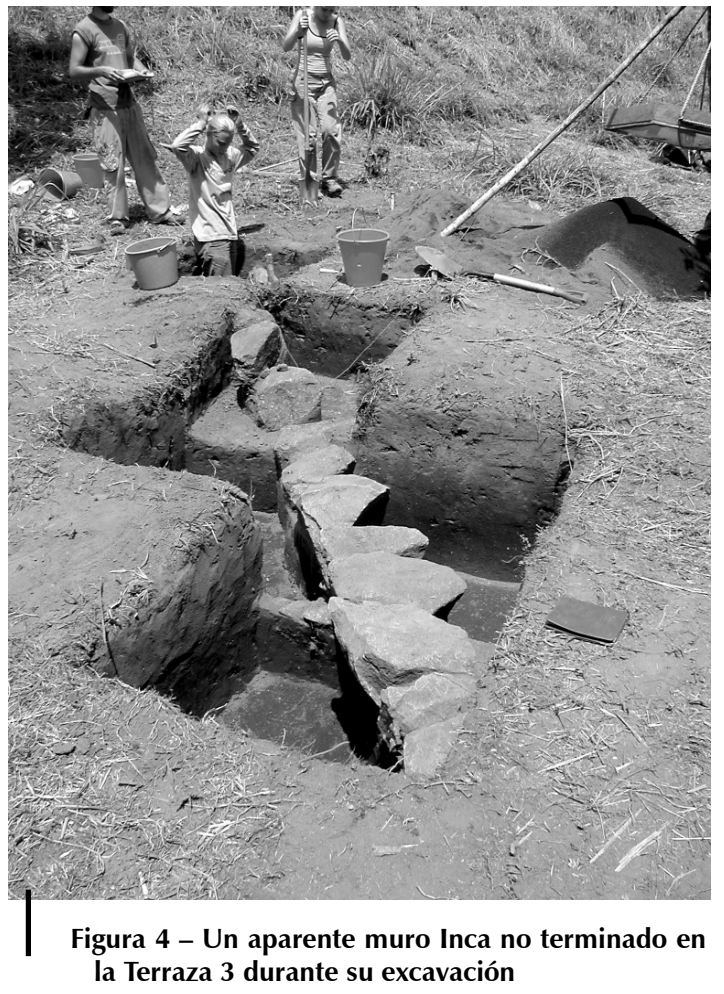

Se ve en esta foto aproximadamente la mitad de lo que se descubrió
En el año 2003 se cortó la caña de azúcar en el terreno justamente debajo del Área A. Se aprovechó la limpieza y realizamos casi cien pruebas de pala en este terreno de aproximadamente dos hectáreas (designada «Área B»). Se recuperó cerámica en todas las pruebas menos una y, en general, fue una mezcla de alfarería de Yumbos e Incas. Luego excavamos una serie de sondeos de $1 \mathrm{~m} \times 1 \mathrm{~m}$ en el mismo terreno encima de tres pequeñas terrazas que podrían ser naturales o artificiales. Se pudo determinar que la densidad de cerámica era mayor en el Área B que en el Área A. Estas concentraciones cerámicas y las construcciones de piedras nos permitieron suponer que el Área A fue empleada mayormente para funciones cívicas, administrativas o religiosas.

En 2006 el gobierno provincial construyó una cancha de fútbol en la parte baja de la loma («Área D»), ubicada más abajo del Área B. A pesar de que toda este área era parte del sitio arqueológico, no pudimos detener su destrucción cuando

los tractores aplanaron esta sección de la loma. Algunos moradores recolectaron piezas que ahí surgieron. Gracias a este esfuerzo, pudimos corroborar que el sitio seguía hasta el pie de la loma y llegaba al pueblo actual. No fue posible definir los límites del sitio arqueológico. Esto no era sorprendente ya que se sabía que los pueblos yumbos no fueron asentamientos nucleados sino dispersos (Salomon, 1997: 33). No obstante, nuestra conclusión preliminar fue que la loma sirvió como centro administrativo con limitado uso doméstico mientras la mayor parte de la población habitaba las partes más bajas sobre la meseta donde hoy día se asienta el pueblo de Palmitopamba.

Las excavaciones preliminares del sitio se han llevado a cabo en tres temporadas, entre 2002 y 2004. En 2003 Tamara Bray se separó del proyecto y Alejandra Gudiño la reemplazó como codirectora, cargo que desempeña hasta el presente. Con los hallazgos mencionados arriba y otros no puntualizados aquí, podemos confirmar la existencia en el sitio de una ocupación Yumbo de larga duración, aproximadamente desde 1100 d. C. hasta el siglo XVI, y también de una ocupación Inca y Yumbo al final de ese periodo. Más adelante hablaremos un poco más sobre la datación de la ocupación Inca pero por el momento basta decir que se trata de un episodio relativamente corto a principios del siglo XVI. De igual manera nos ha sido posible comprobar que los yumbos contaban con una organización 
sociopolítica lo suficientemente compleja como para modificar el paisaje de Palmitopamba, creando un centro regional importante y estratégico. También queda bastante claro que los incas que llegaron a Palmitopamba se asentaron conjuntamente con los yumbos, aparentemente sin contiendas significantes. Se escribirá sobre esta relación Yumbo-Inca más adelante.

Suspendimos las excavaciones en 2005 para emprender, junto con la comunidad, los trabajos de puesta en valor del sitio arqueológico. En 2006 el geofísico Donald Johnson aceptó nuestra invitación de volver al sitio, esta vez con un instrumento de radar subterráneo (ground-penetrating radar). En las terrazas 1-3 y 5-6 no se reportaron mayores anomalías pero en la Terraza 4, que era la terraza más pequeña y más baja del Área A, se descubrieron varias anomalías. Por lo tanto volvimos al sitio en 2007 con el objetivo de investigar estas anomalías de la Terraza 4. A continuación se brinda una descripción de lo encontrado.

\section{EXCAVACIONES EN LA TERRAZA 4 DESDE 2007}

El primer descubrimiento de la temporada fue un muro de retención parcialmente demolido en el límite occidental de la terraza. Creemos que este muro se conectaba con el muro de contención de la Terraza 3, hipótesis que esperamos confirmar en la temporada de 2010.

En la parte central de la Terraza 4 empezaron a aparecer muchas agrupaciones de piedras. El hallazgo de estas concentraciones aparentemente sin ningún patrón y con funciones desconocidas nos dejó al principio bastante confundidos. A mitad de la temporada encontramos un fragmento de un muro sin lugar a dudas de manufactura Inca y que probablemente pertenecía a un edificio Inca. Después de dos temporadas de trabajo, supimos que el edificio era rectangular, que sus muros tenían $80 \mathrm{~cm}$ de grosor, y que medían en el exterior 7,1 $\mathrm{m}$ de largo y 5,6 m de ancho (fig. 5). En general se emplearon dos tipos diferentes de piedra. Una parecía ser una granodiorita dura que fue labrada y careada, empleada en las construcciones de las caras exteriores de los muros. La otra era una piedra desmenuzable simplemente conocida como cascajo, utilizada como relleno al interior de los muros.

Más precisamente, sabemos que no se trata de un edificio sino de los cimientos de un edificio. Una de las posibilidades es que en este edificio hubieran cimientos de piedra que sirvieran de soporte a una construcción de adobe o de madera o caña. Sin embargo, las evidencias parecen favorecer la hipótesis de un edificio de piedra incompleto en una etapa temprana de la construcción. Por ejemplo, la existencia de un segundo muro alrededor del muro principal, que solamente llega a extenderse a la mitad, permite pensar en una construcción abandonada. La presencia de amontonamientos de materiales de construcción incluyendo piedra sin labrar, piedras labradas y arcilla amarilla que puede haber servido de mortero podría reforzar esta hipótesis del abandono. También se ha considerado la posibilidad de que en efecto el edificio se hubiera terminado y desmantelado en un periodo posterior, quizás para aprovechar las piedras en otra construcción. 


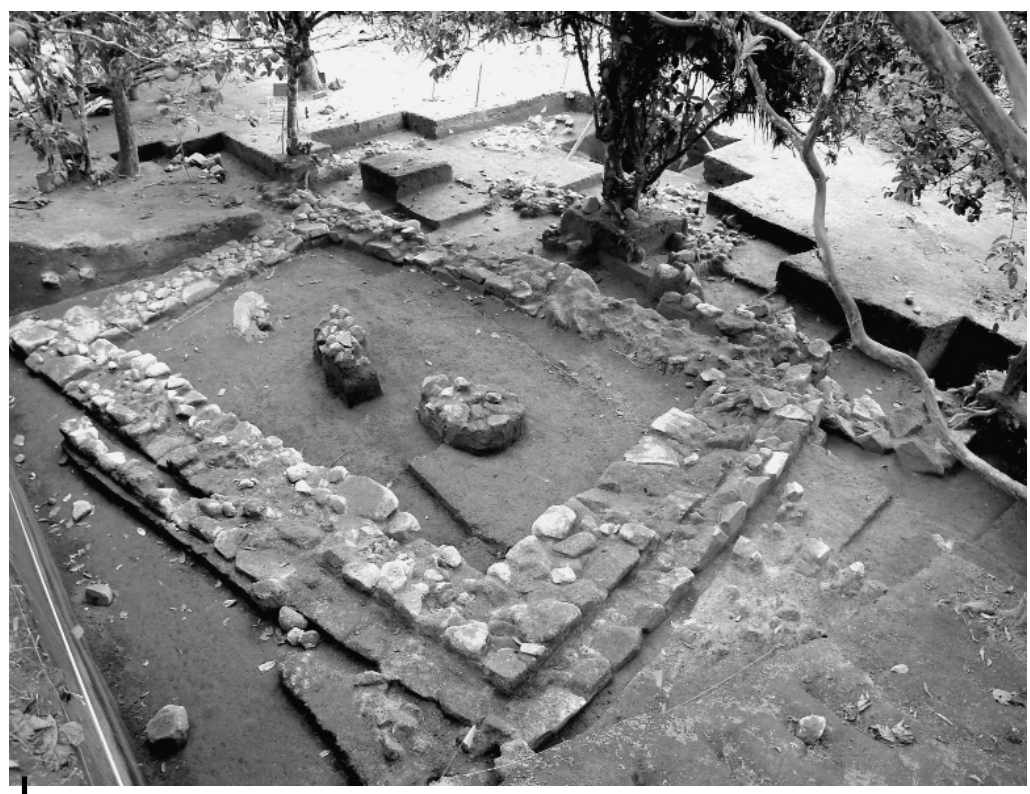

Figura 5 - El edificio Inca en la Terraza 4 y varios rasgos de piedra que pertenecen a la misma ocupación

La única ocupación posterior que se conoce comienza en el siglo XX cuando el edificio ya estaba enterrado e invisible, lo cual hace de esta una hipótesis poco probable.

Otros rasgos en el área del edificio comprenden muros muy erosionados, un área con una especie de pavimento de piedra y una hoguera de $1 \mathrm{~m}$ de diámetro. Además, el edificio está rodeado de varias concentraciones de piedras, algunas de las cuales son cuidadosamente construidas mientras que otras parecen ser depósitos de materiales de construcción.

A pocos metros del edificio Inca y propio de la misma ocupación, encontramos dos concentraciones de piedras casi iguales, una al lado de la otra (fig. 6). Cada una es de forma ovalada u oblonga con casi $2 \mathrm{~m}$ de largo y un poco menos de $1 \mathrm{~m}$ de ancho. En 2008 se decidió excavar la mitad de uno de estos rasgos pensando que las piedras podrían marcar un enterramiento. Fue sorprendente encontrar que este rasgo alcanzaba una profundidad de 1, $5 \mathrm{~m}$ y el relleno de piedras conservaba la forma ovalada y el mismo tamaño que se encontró en la superficie. No se han recuperado artefactos con la excepción de un solo tiesto de loza colonial, una cerámica muy rara en el sitio. Se decidió entonces excavar la otra mitad del rasgo. Para mayor sorpresa la forma y el tamaño del óvalo en que las piedras habían sido acomodadas desde la superficie se mantenía y no se encontró ninguna evidencia de entierro. Investigando, se encontró un reporte de Idrovo Urigüen (2000: 272) para Tomebamba en el sur del Ecuador en donde se mencionaban rasgos de piedras muy similares. Se pudo comparar también estos 


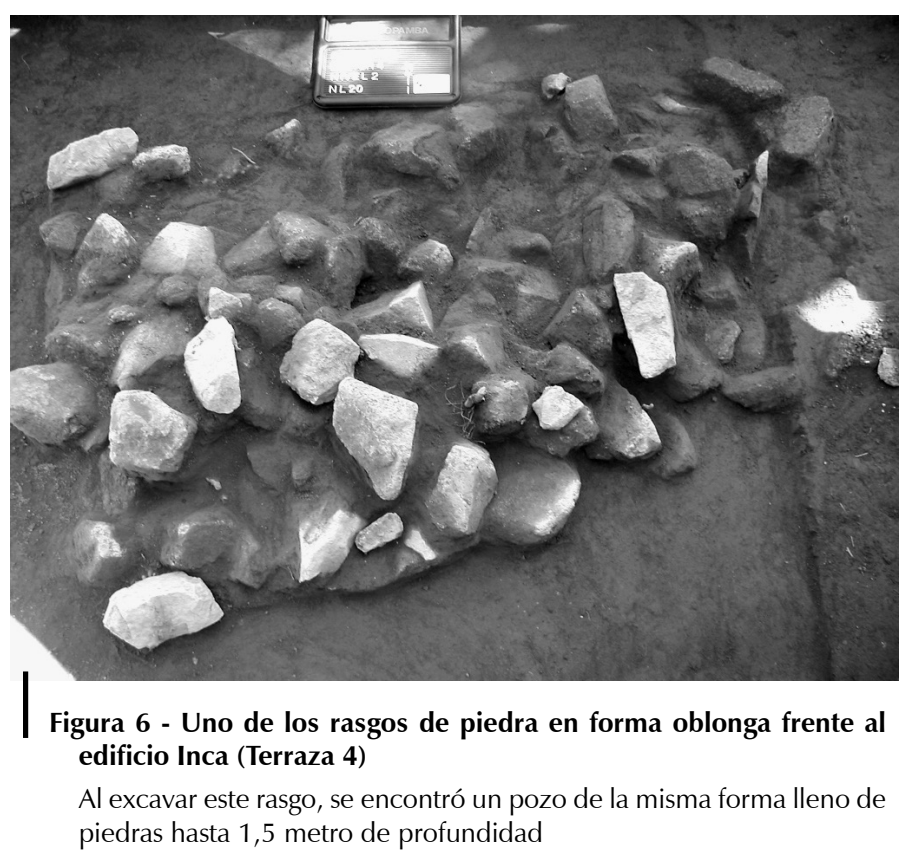

rasgos con uno recientemente excavado por Bray (2009, comunicación personal) en Caranqui en la sierra norte del Ecuador. Ambos son sitios Incas muy importantes ocupados por el emperador. En ambos casos los rasgos resultaron ser entierros. En el caso de Tomebamba, el entierro no se encuentra al fondo del pozo y debajo de las piedras sino en una pequeña cámara lateral. Con esta información, en 2009 se excavó un área mayor alrededor del rasgo para buscar un entierro pero no lo se encontró. Pensamos que estos rasgos podrían ser tumbas falsas para despistar a los saqueadores de la época, una táctica utilizada en Egipto para proteger algunas tumbas de lujo.

Lo que sí se encontró fue otro muro Inca paralelo a los muros largos del edificio pero a una distancia de $6 \mathrm{~m}$ (fig. 7). Este muro, que mide aproximadamente $8,5 \mathrm{~m}$ de largo (aunque es posible que sea más largo y que aún no hayamos encontrados los extremos) y $50 \mathrm{~cm}$ de ancho, se ubica cerca del borde de la terraza. Todo el conjunto de rasgos en la Terraza 4 dan la impresión de una cancha en el sentido andino, es decir un complejo de edificio y patios rodeados por un muro.

\section{NL-18-CEMENTERIO YUMBO DE PALMITOPAMBA}

En la parte oriental del pueblo de Palmitopamba hay una colina baja donde se ubica un cementerio Yumbo caracterizado por una serie de tolas redondas pequeñas, o túmulos. Lippi descubrió en 1984 esta concentración de tolas y la registró como NL-18 pensando que se trataba de un nuevo sitio. Posteriormente se pudo determinar que NL-20 era grande y abarcaba el área de NL-18. Es decir, 


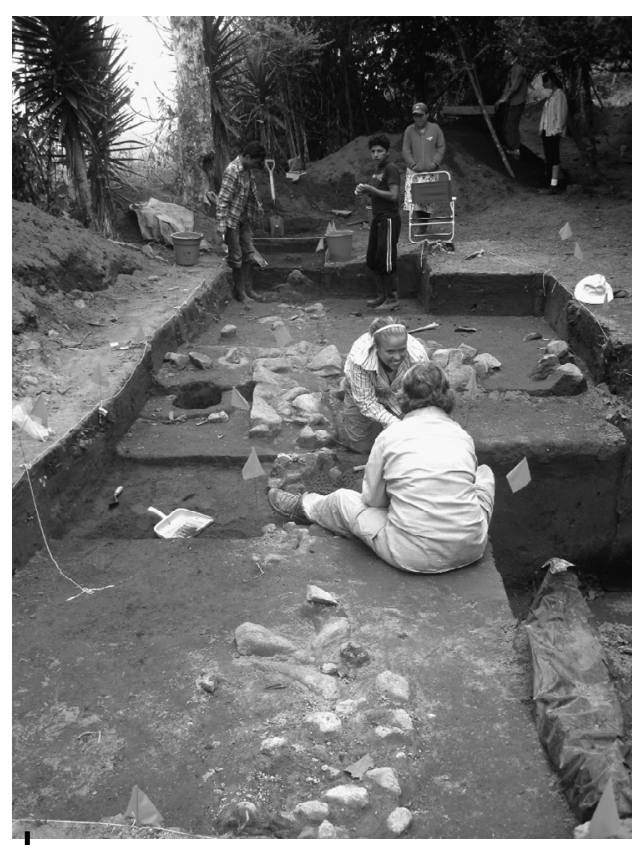

Figura 7 - El muro en la Terraza 4 que se descubrió en 2009 frente al edificio Inca y paralelo al eje mayor del edificio

La mayor parte no está bien conservado o nunca fue terminado el sitio monumental y el cementerio forman parte del mismo asentamiento antiguo. Con la excepción de un par de excavaciones casuales hechas por moradores, el cementerio no corría peligro, de manera que por varios años no fue una prioridad excavar allí. Sin embargo, en 2006, un pariente del propietario dijo su interés en cavar una de las tumbas, lo cual nos causó alarma. Hasta ese entonces no existía ninguna excavación sistemática y profesional realizada por arqueólogos en una tumba Yumbo, aunque se sabía de algunos cementerios Yumbos saqueados por buscadores de tesoros. En 2007 fue seleccionado un túmulo en la cima de la colina para el estudio. Estos túmulos miden aproximadamente $4 \mathrm{~m}$ de diámetro y $1,5 \mathrm{~m}$ de altura sobre la superficie actual del terreno. Hasta el momento no se ha podido determinar el número exacto de túmulos en el área del cementerio ya que la mayor parte se encuentra cubierto por un cañaveral.

En el primer túmulo, designado Tola 1, se abrió un corte de $2 \mathrm{~m} \times 2 \mathrm{~m}$ en busca de la «boca» de la tumba, la cual apareció indistintamente a una profundidad de 1,20 m bajo la parte más alta de la tola. Hasta los $2 \mathrm{~m}$ de profundidad, la fosa estaba claramente definida. La fosa tenía un largo de casi $2 \mathrm{~m}$ indicando que se trataba de un entierro extendido. A los 2,10 $\mathrm{m}$ de profundidad se encontraron tres vasijas —un cántaro y dos platos en pedestal- . Luego apareció el cráneo en estado avanzado de descomposición. Las tres vasijas estaban ubicadas cerca de la cabeza del difunto (fig. 8). También apareció el hueso de la mandíbula con un diente todavía en su sitio que tuvo suerte de una mejor preservación acompañado por varios dientes sueltos. Un análisis muy preliminar de los dientes indicó que se trataba de un subadulto. La mandíbula presentaba un color verduzco por la presencia de un collar con artefactos de cobre. Lo único que se pudo rescatar del resto del esqueleto fueron pequeños fragmentos de vértebras y de otros huesos no identificables.

Se encontraron dos collares, uno de numerosas cuentas muy pequeñas de concha Spondylus y otro con cuentas cerámicas aún ensartadas sobre un alambre de cobre. En el mismo collar se encontraron dos pequeños cascabeles de cobre y un colgante del mismo metal. Un análisis metalúrgico de estos artefactos en el Laboratorio de Química del Instituto Nacional de Patrimonio Cultural (Romero, 2008) en Quito confirmó que se trataba de cobre puro. Pegado a uno de los cascabeles se había preservado un nudo de fibra. Según el análisis del mismo laboratorio, se trataba de fibra de algodón con las hebras de torsión Z y los hilos de torsión $\mathrm{S}$, con el cordón formado por cuatro hilos formando posiblemente un 


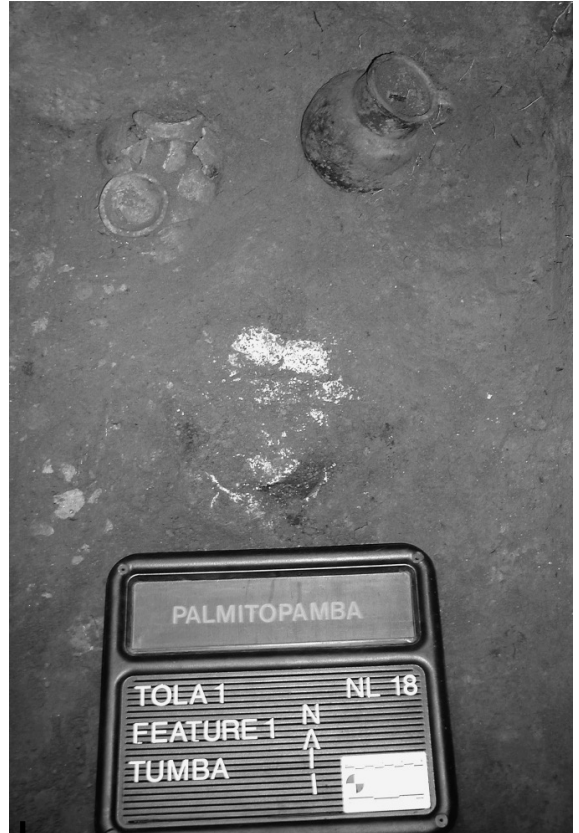

Figura 8 - Los primeros indicios del entierro en la Tola 1 (NL-18)

Arriba a la izquierda están dos platos rotos, a la derecha un cántaro, y en la mitad se ve el hueso casi desintegrado del cráneo nudo sobre el cascabel. Pegado al otro cascabel se encontró un pequeño fragmento de textil. El mismo laboratorio (Romero, 2008) determinó que el fragmento de casi $2 \mathrm{~cm} \times 2 \mathrm{~cm}$ tenía las mismas características que el nudo sobre el cascabel pero con ocho fibras de trama y ocho de urdimbre por centímetro. La estructura era un tafetán simple (con un solo hilo de trama que cruzaba con uno de urdimbre).

Durante la excavación de la tumba, se registró la presencia apenas visible de un pigmento rojo en las paredes. Se encontraron en la tumba unas pocas piedras pequeñas con pigmento rojo. El Laboratorio de Química del INPC (Romero, 2008) analizó el pigmento y determinó que era hemetita $\left(\mathrm{Fe}_{2} \mathrm{O}_{3}\right)$. Creemos que las piedras con pigmento se utilizaron para preparar la tumba con colores.

En 2008 se tomó la decisión de excavar otra tola (Tola 2) justamente al lado de la Tola 1 y de la misma forma y tamaño. Se utilizó el mismo método para ubicar la boca de la tumba, la cual apareció como un cuadrado de casi $1 \mathrm{~m} \times 1 \mathrm{~m}$ a $125 \mathrm{~cm}$ debajo de la parte alta de la tola. Obviamente se trataba de otro tipo de entierro que el de la Tola 1. En efecto la tumba que comenzó como un cuadrado disminuía de tamaño conforme profundizábamos la excavación de la fosa. La primera evidencia del entierro apareció a 2,20 m bajo la superficie (la parte alta del túmulo). Lo primero que se descubrió fue una pulsera de cobre. Luego apareció un brazo con otra pulsera de cobre en la muñeca. Debajo del brazo encontramos el cráneo con dos aretes de cobre y con un disco cerámico de aproximadamente $8 \mathrm{~cm}$ de diámetro, que pudo haber sido un plato labial o quizás un pequeño platito para incienso (fig. 9). Debajo del cráneo y en forma fetal o flexionado, se descubrió el resto del esqueleto en un estado de conservación bueno, comparado con el esqueleto mayormente desintegrado excavado durante la temporada anterior y tomando en cuenta que estamos en una zona de bosque tropical.

En el fondo del pozo a $240 \mathrm{~cm}$ bajo la superficie, encontramos cuatro vasijas quebradas pero mayormente enteras (fig. 10). Dos de éstas son de formas clásicas Incas («beaker» y aríbalo) mientras que las otras dos son platos Yumbos o de la Sierra Norte. Aún no se hace el análisis definitivo porque las vasijas están siendo reconstruidas por un especialista. El hecho de encontrar artefactos de los incas en una tumba supuestamente Yumbo en un cementerio que definitivamente era de los yumbos fue extraordinario. Sin embargo, esta evidencia apoya la hipótesis que se brinda más adelante y que sugiere que los incas se refugiaron en el País Yumbo, 


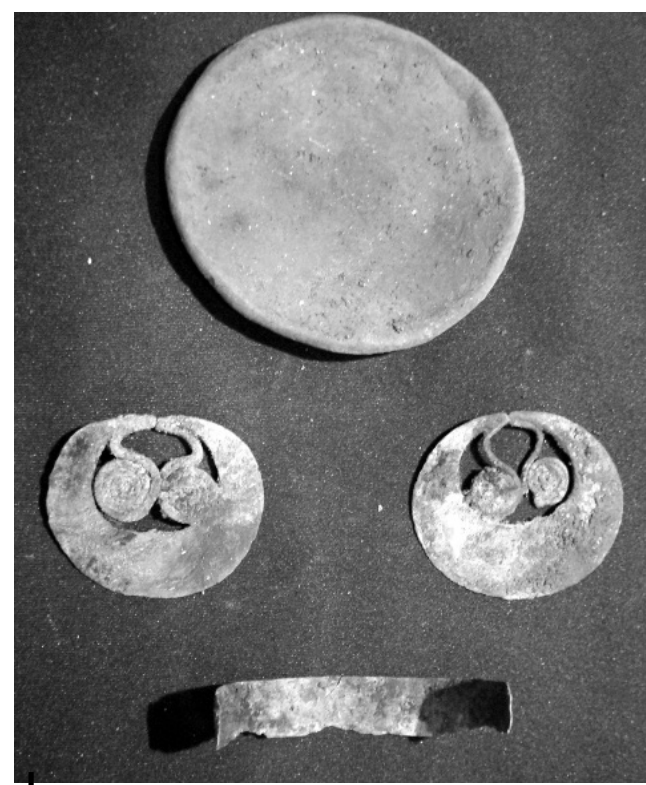

Figura 9 - Cuatro artefactos asociados al muerto en la Tola 2 (NL-18)

Arriba está un disco cerámico, en la mitad dos aretes de cobre (posiblemente con platino), y una de las dos pulseras de cobre que convivieron pacíficamente y que hubo un mestizaje de los dos grupos.

\section{DATACIÓN DEL SITIO}

La datación del sitio de Palmitopamba se hizo principalmente a partir del carbono 14. Hasta el momento se cuenta con dieciséis fechas radiocarbónicas calibradas (método AMS). La mayoría de las dataciones son de residuos quemados de comida pegada en ollas, lo cual disminuye notablemente la posibilidad de un contexto falso para el carbón. Con la excepción de una muestra que produjo una fecha muy temprana e inexplicable de $2070 \pm 50$ antes del presente (a.p.), todas definen un periodo de ocupación del sitio que va aproximadamente desde 1000 d. C. hasta 1620 d. C. El comienzo coincide más o menos con la datación vulcanológica de la tefra de Quilotoa, que según Patricia Mothes (comunicación personal, 2005) es de 850 a. p. (no calibrada), lo que viene a ser entre 1046 y

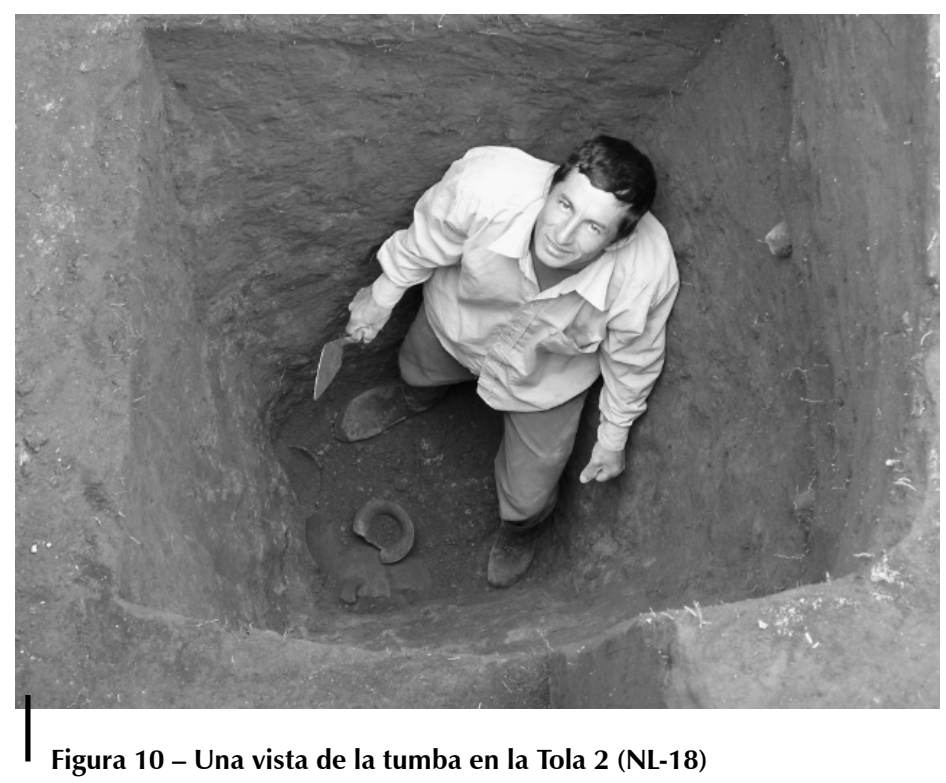

Se ven pedazos de un aríbalo Inca que estuvo al fondo de la tumba debajo del muerto 
1267 d. C. (calibrada). Esto nos permite concluir que los yumbos estuvieron en el sitio y construyeron las terrazas antes de esta erupción.

Después de establecer el periodo aproximado para la ocupación Yumbo, nos interesó determinar la fecha de la llegada de los incas a Palmitopamba. Desafortunadamente, la curva de calibración para fechas radiocarbónicas alrededor de 1500 d. C. es bastante imprecisa y no permite determinar esta fecha con la minuciosidad deseada. Se hace inprescindible la etnohistoria para refinar este marco cronológico. Los recuentos etnohistóricos indican que los incas mantuvieron una guerra contra los caranquis en la Sierra Norte del Ecuador durante una década a finales del siglo XV, es decir alrededor de 1490 a 1500 d. C. El contacto Inca-Yumbo posiblemente se inició ya que los señoríos serranos mantenían contacto continuo con los yumbos para intercambiar productos de la montaña tropical con los de la sierra (Salomon, 1997: 18-19). Así, la llegada de los incas a Palmitopamba pudo haber sucedido un poco antes de 1500 y es probable que duró hasta después de la conquista española en 1532. Es de recordar que la entrada de Benalcázar y sus tropas al Ecuador se reportó para 1534. Hasta el momento no se pudo confirmar esta hipótesis por medio de la datación radiométrica. Sin embargo, Salomon (1997: 25-26) encontró documentos sobre incas que buscaron refugio en el País Yumbo después de la Conquista y, por razones que explicaremos más adelante, se piensa que Palmitopamba pudo haber recibido a un contingente Inca importante en 1534.

Una de las dieciséis fechas radiocarbónicas pertenece a la tumba de Tola 2 excavada en el cementerio vecino de NL-18. La datación calibrada de esta tumba que contiene elementos Incas tanto como Yumbos va desde 1430 a 1530 d.C.

Se encontró muy poca cerámica de loza colonial en el sitio y no se pensó que hubo mayor contacto con los españoles debido a la falta casi total de evidencias. En otras palabras, Palmitopamba fue un escondite pero no se pudo determinar aún por cuántos años. No existió ninguna evidencia de una ocupación posterior en el sitio hasta el siglo XX cuando un par de colonos mestizos se asentaron sobre la meseta y crearon dos haciendas que luego fueron lotizadas y vendidas a otros moradores más recientes, fundando de esta manera el pueblo actual de Palmitopamba.

\section{RUMIÑAHUI}

En 2003 la historiadora ecuatoriana Tamara Estupiñán Viteri publicó un libro sobre el líder indígena Rumiñahui, quien fue el comandante del ejército Inca (un «general» si uno quiere asignarlo un rango más alto) encargado de la guardia personal del Sapa Inca Atahualpa. Rumiñahui fue un líder muy polémico en la historiografía y también en la política andina actual. Los peruanos y otros partidarios de Húascar consideraron que Rumiñahui fue un traidor al Tahuantinsuyu y los ecuatorianos lo consideraron el mayor héroe de la resisitencia indígena contra los españoles. Más allá de esta polémica, Estupiñán Viteri reunió documentos etnohistóricos que indicaban que Rumiñahui, después del secuestro y ejecución de Atahualpa 
por Francisco Pizarro, obtuvo el cadáver de Atahualpa y huyó de Quito con sus restos mortales, algunas esposas, herederos y pertenencias personales de Atahualpa, y posiblemente con una parte del tesoro que no se entregó a Pizarro. Rumiñahui con este contingente real fue al País Yumbo donde mandó a construir un fuerte. Todo parece indicar que los españoles bajo el comando de Ruy Díaz fueron capturados en el País Yumbo y sus tesoros supuestamente saqueados. Posteriormente Rumiñahui viajó a la sierra liderando un levantamiento contra los españoles que fracasó y fue asesinado sin poder consolidar la resistencia indígena contra los españoles.

Desde nuestra perspectiva, el dato más interesante entre toda la polémica y las varias interpretaciones sobre los acontecimientos y las motivaciones era la declaración de que Rumiñahui construyó un fuerte en el País Yumbo (Estupiñán Viteri, 2003). A través de las prospecciones a nivel regional, Lippi pudo confirmar la existencia de cuatro pucaras Incas en el País Yumbo y documentó la posibilidad de algunos más que no fueron confirmados. Entre éstos el fuerte más grande y aparentemente más importante fue el de Palmitopamba. Hay otros sitios con presencia Inca importantes en la región entre los cuales nombramos el pucara de Chacapata, el tambo de Capillapamba, y el complejo de piscinas de Tulipe. Hasta el momento los descubrimientos en Palmitopamba fueron los que más llamaron la atención como un posible refugio Inca y como el posible fuerte que Rumiñahui construyó.

Para apoyar esta hipótesis, hay que confirmar la presencia Inca en territorio Yumbo y particularmente en Palmitopamba alrededor de 1534, época en que Rumiñahui supuestamente buscaba refugio en el País Yumbo con los herederos de Atahualpa. Es de gran importancia a su vez descubrir en Palmitopamba evidencias contundentes de la presencia de la realeza Inca o descartar la misma en los otros sitios que son candidatos. Si este asunto de identificar el paradero de Rumiñahui no parece tener tanta importancia, basta recalcar el estatus de Rumiñahui en el Ecuador hoy en día y también la necesidad de recuperar datos históricos sobre el levantamiento contra los españoles en el norte del Ecuador.

\section{ALGUNAS INTERPRETACIONES PROVISIONALES SOBRE LA RELACIÓN INCA-YUMBO}

Se ha llegado a comprender que Palmitopamba fue un centro importante de los yumbos y que los incas se apoderaron del sitio de una manera aparentemente no belicosa. Se supone que la interacción entre yumbos e incas comenzó durante la expansión hacia el norte del Tahuantinsuyu y que los yumbos sirvieron como productores y comerciantes de bienes tropicales muy cotizados por los incas igual como fueron para las etnias locales antes de la llegada de los incas al Ecuador septentrional. Ésta fue una posibilidad. Otra posibilidad, que no es necesariamente exclusiva de la anterior, fue que los incas enviaron tropas al País Yumbo durante la alargada guerra contra los caranquis al norte de Quito para defender el flanco occidental. En cualquier caso, hay que comprobar la presencia de los incas en el sitio alrededor de 1490 ó 1500, prueba que aún no existe. Igualmente podría ser 
que los incas no estuvieron en Palmitopamba hasta finales del imperio cuando fue ejecutado Atahualpa y cuando la nobleza quiteña se refugió en el País Yumbo. Si esto ocurrió bajo el liderazgo de Rumiñahui, no se puede acertar todavía este asunto. La presencia Inca en Palmitopamba pudo haber comenzado por una razón u otra tres o cuatro décadas antes de la llegada de los españoles, o pudo haber empezado alrededor de 1534 con la caída súbita del Tahuantinsuyu. O pudo haber sido una presencia continua o esporádica que abarcó ambos sucesos en la historia antigua del Ecuador. Obviamente, hay mucha incertidumbre hasta obtener una cronología más minuciosa.

No obstante la cronología inexacta, las evidencias del sitio señalan una relación intrigante entre los autóctonos y los invasores. Primero, los incas aparentemente respetaron la tola yumba encima de la loma. Segundo, en la mayor parte del sitio, menos en la tola, se ve una mezcla de cerámica Inca y cerámica Yumbo durante la fase más tardía de ocupación del sitio. Aunque se puede diferenciar fácilmente entre vasijas Yumbos e Incas por su forma y decoración, se han obtenido resultados de análisis a base de activación de neutrones (Speakman \& Glascock, 2003) 5 y resulta que toda la cerámica (con la excepción de algunos pocos tiestos de la tradición Cosanga o Panzaleo) es hecha de la misma pasta local. Es decir, es casi seguro que la cerámica Inca es de elaboración local probablemente por las mismas alfareras yumbas. Tercero, hay una buena probabilidad de que los incas se hayan asentado en Palmitopamba al menos en parte para obtener recursos y productos del bosque tropical y que la relación entre los dos haya sido mayormente económica. Es posible que la relación establecida años atrás haya sirvido para dar a los incas la confianza de refugiarse entre los yumbos y construir un fuerte como base para organizar la resistencia contra los españoles.

Si en efecto nobles o hasta miembros de la familia real de los incas se refugiaron entre los yumbos, aún después de la salida de Rumiñahui, el levantamiento fracasado y su muerte, una población de tamaño no determinado de incas aparentemente se quedó en la zona. La existencia de una tumba con vasijas Incas en un cementerio Yumbo de Palmitopamba nos hizo pensar que hubo un «mestizaje» de nativos e invasores en el País Yumbo. A pesar de la falta de evidencias contundentes para apoyar o no estas varias hipótesis, parece inevitable la conclusión de que la interacción entre yumbos e incas en Palmitopamba fue idiosincrática y que merecía más estudio para comprender mejor la expansión y el mantenimiento del Tahuantinsuyu.

\section{LA PUESTA EN VALOR DEL SITIO}

Existe un compromiso con el pueblo de Palmitopamba. Se han brindado al pueblo algunos beneficios entre los cuales se puede incluir lo siguiente: trabajo en este pueblo con una alta tasa de desempleo, ingresos para comerciantes durante

5 Muestras adicionales analizadas en el mismo laboratorio en 2004, 2005 у 2009 confirmaron los resultados de 2003. 
nuestra estadía, y conocimientos sobre la arqueología del sitio de Palmitopamba y la historia muy temprana de la zona. Ellos ofrecen trabajadores inteligentes y entusiastas y ayudan a proteger el sitio contra los huaqueros y las fuerzas de la naturaleza. También reciben con respeto y amabilidad a los investigadores. Por medio de la generosidad de la Butler Foundation' de los EE.UU., que es fuente del financiamiento para realizar el Proyecto Arqueológico Palmitopamba, en los últimos años se ha podido ayudar economícamente a la comunidad para llevar a cabo proyectos para el mejoramiento del pueblo, como son el nuevo sistema de agua potable, un muro ornamental alrededor del parque, y apoyo para el establecimiento de una tienda comunitaria. Por lo tanto, se ha podido establecer una relación de beneficio mutuo durante las distintas temporadas de excavación desde 2002.

Con el interés que despertó el hallazgo en el pueblo mismo y la evidencia de que se trata de un sitio arqueológico de mucha importancia, se ha llegado con los moradores al deseo de adecuar el sitio para recibir al público. El fin de este proyecto es de educarlos sobre la historia antigua del sector y la cultura desaparecida de los yumbos, sobre su actuación intrigante en el Tahuantinsuyu, y luego su posible papel como refugio para los incas reales que se escondieron de los españoles. En esta zona tan bella pero poco desarrollada, el parque arqueológico podrá servir como punto clave para un desarrollo ecoturístico en general. La idea es de entregar al pueblo el sitio y la mayor responsabilidad para su manejo como parque arqueológico, contando con los autores de esta investigación como consultores arqueológicos. La puesta en valor del sitio es un proyecto ambicioso que se espera llevar a cabo poco a poco en los próximos años. Depende de otros avances como la pavimentación del camino que llega a Palmitopamba, la construcción de una infraestructura básica para turistas, la entrega de las propiedades dentro del sitio arqueológico a la comunidad, y otros desafíos. Sin embargo, se debe reconocer el apoyo que brindó el pueblo durante varios años y hasta cierto punto reciprocarlo en este sentido.

\section{Referencias citadas}

CABELLO VALBOA, M., 1951 [1586] - Miscelánea antártica. Una historia del Perú antiguo, 561 pp.; Lima: Universidad Nacional Mayor de San Marcos. Instituto de Etnología, Seminario de Historia del Perú-Incas.

ESTUPIÑÁN VITERI, T., 2003 -Tras las huellas de Rumiñahui, 162 pp.; Quito: Auspicio del Banco General Rumiñahui.

IDROVO URIGUEN, J., 2000 - Tomebamba: Arqueología e Historia de una Ciudad Imperial,

6 Agradecemos a la Butler Foundation de Concord, New Hampshire, EE.UU., por su apoyo generoso del Proyecto Arqueológico Palmitopamba durante varios años. 
348 pp.; Cuenca: Banco Central del Ecuador.

LIPPI, R. D., 1998 - Una exploración arqueológica del Pichincha Occidental, Ecuador, 372 pp.; Quito: Museo Jacinto Jijón y Caamaño de la Pontificia Universidad Católica del Ecuador, Consejo Provincial de Pichincha.

LIPPI, R. D., 2001 - Yumbo demographics before and after 1532. Paper presented at the $29^{\text {th }}$ Annual Midwest Conference of Andean and Amazonian Archaeology and Ethnohistory, University of Michigan, Ann Arbor (EE.UU.).

LIPPI, R. D., 2004a - Tropical forest archaeology in Western Pichincha, Ecuador, 168 pp.; Belmont, California: Case Studies in Archaeology, Jeffrey Quilter, series editor, Thomson/Wadsworth.

LIPPI, R. D., 2004b - La expansión de las poblaciones barbacoas en el noroeste de Ecuador. Revista de Arqueología del Área Intermedia, 6: 249-276; Bogotá: Instituto Colombiano de Antropología e Historia y Sociedad Colombiana de Arqueología.

LIPPI, R. D. \& GUDIÑO, A.M., 2010 - Inkas and Yumbos at Palmitopamba in northwestern Ecuador. In: Distant provinces in the Inka empire: Toward a Deeper Understanding of Inka Imperialism (M. Malpass \& S. Alconini, eds.): 260-278; lowa City: University of lowa Press.

ROMERO, M., 2008 - Informe de resultados (análisis composición química, análisis de textiles y análisis de estrato pictórico en piedra); Quito: Laboratorio de Química, Instituto Nacional de Patrimonio Cultural.

SALOMON, F., 1997 - Los Yumbos, Niguas y Tsáchila o «Colorados» durante la colonia Española: etnohistoria del noroccidente de Pichincha, Ecuador, 131 pp.; Quito: Ediciones Abya-Yala.

SPEAKMAN, R. J. \& GLASCOCK, M. D., 2003 - Instrumental neutron activation analysis of ceramics and clays for the Palmitopamba Archaeology Project, Ecuador, 17 pp.; Columbia: Missouri University Research Reactor, University of Missouri, 31 January 2003. 